\title{
Platelet-Rich Plasma Diffusion in Achilles Tendon: Relationship with Therapeutic Outcomes
}

\author{
Michele Abate Luigi Di Carlo Vincenzo Salini \\ Department of Medicine and Science of Aging, University G. d'Annunzio, Chieti-Pescara, Chieti Scalo, Italy
}

\section{Significance of the Study}

- It is not known whether the therapeutic efficacy of platelet-rich plasma in the treatment of Achilles tendinopathy can be ascribed to its distribution inside the tendon. In the present study, no relationship was found between plasma diffusion and clinical outcomes. Therefore, the peppering technique, which allows the distribution of plasma over a wide region of a tendon considered as a functional unit, is recommended.

\section{Keywords}

Achilles tendon · Platelet-rich plasma · Outcome

\begin{abstract}
Objective: The therapeutic efficacy of platelet-rich plasma in the treatment of Achilles tendinopathy is controversial. Among the variables which can explain the discrepant results, little attention has been paid to the distribution of platelet-rich plasma inside the tendon. The aim of the present study was to evaluate whether this factor is related to the clinical outcome of treatment. Subjects and Methods: Forty patients suffering from mid-portion Achilles tendinopathy were studied. At baseline, pain and function were measured by means of a visual analogue scale and the Victorian Institute of Sport Assessment-Achilles questionnaire. Thereafter, an ultrasound examination was performed, and platelet-rich plasma was injected. Immediately after injection, the plasma distribution was assessed visualizing the material in the tendon. The patients were re-evaluated at 3 and 6 months, computing pain and function values and the percentage of sat-
\end{abstract}

\begin{tabular}{ll}
\hline KARGER & $\begin{array}{l}\text { (c) 2019 The Author(s) } \\
\text { Published by S. Karger AG, Basel Openger }\end{array}$ \\
E-Mail karger@karger.com & $\begin{array}{l}\text { This is an Open Access article licensed under the Creative Commons } \\
\text { Attribution-NonCommercial-4.0 International License (CC BY-NC) } \\
\text { (http://www.karger.com/Services/OpenAccessLicense), applicable to } \\
\text { the online version of the article only. Usage and distribution for com- } \\
\text { mercial purposes requires written permission. }\end{array}$
\end{tabular}

isfactory outcomes in relation to the distance reached by the plasma from the point of injection. Results: The diffusion inside the tendon was longitudinal in all cases and cross-sectional only in 9. The mean distance from the tip of the needle increased significantly according to the severity of tendon damage. At follow-up, no relationship was found between plasma diffusion and clinical outcomes. Conclusions: Platelet-rich plasma diffusion has no effect on clinical outcome, which mainly depends on the metabolic activation of the whole structure of the tendon. @ 2019 The Author(s) Published by S. Karger AG, Basel

\section{Introduction}

The therapeutic efficacy of platelet-rich plasma (PRP) in the treatment of tendinopathies is still controversial. In some cases, complete healing was observed, in others the efficacy was poor and short-lasting, or no benefit at all was reported $[1,2]$. Several factors can explain these discrep- 
ant results; among them, the characteristics of the tendinopathy (the tendon treated and the stage of evolution of the disease) and of the patient (age, sex, genetic background), associated metabolic disorders [3], the individual level of physical activity after treatment and/or the implementation and adherence to rehabilitation, the PRP composition (pure PRP, leukocyte-rich PRP, number of platelets, and content in growth factors), and several other factors (adjuvants, schedule of administration) $[4,5]$.

Among these factors, diffusion of PRP inside the tendons has not been well studied. Studies on adding methylene blue to PRP performed on animal tendon specimens have shown that only part of the product remains in or near the site of injection, whereas a variable amount spreads longitudinally with a prevalent proximal-distal penetration [6]. Similar experiments on human cadaveric Achilles tendons confirmed the longitudinal spreading and also show that PRP can diffuse to the paratenon and the Kager fat pad [7]. However, these studies suffer from important limitations as they were performed in excised or cadaveric specimens and in tendons with a normal structure.

More reliable information comes from experiments performed in vivo on pathologic tendons, exploiting the different PRP and echogenicity of tendon tissues [8-10]. In a retrospective analysis of 52 different tendons (hamstring, elbow, patellar) Loftus et al. [9] observed that the compound, in a large percentage of cases (about 50\%), spread along the paratenon or the tendon sheath and in adjacent soft tissues. Similarly, a prospective study showed that PRP, when injected in elbow tendons, spread to soft tissues outside the tendon in 20 of 39 cases, and the intraarticular extension was observed in 5 cases by Park et al. [10]. There was no difference in the distance of PRP distribution between tendons with and without tears.

A crucial point is whether PRP can have a therapeutic effect even if a large diffusion of the substance occurs far from the site of damage. Therefore, the aim of the present study was to study the diffusion of PRP inside the Achilles tendon and to assess whether it could influence the therapeutic outcomes.

\section{Subjects and Methods}

This study was performed in accordance with the ethical standards as laid down in the 1964 Declaration of Helsinki and its later amendments. Informed written consent for participation was obtained from each patient. Institutional ethics committee approval was not required as this was an observational study [11] and because the PRP procedure is safe and currently performed in routine clinical practice.
Patients suffering from mid-portion Achilles tendinopathy treated with PRP injection were enrolled. The diagnosis was made on the basis of medical history, clinical examination (pain, tenderness, and/or functional limitation), and ultrasound (US) and color Doppler (CD) features of tendon damage.

Exclusion criteria were the following: history of Achilles tendon surgery or tendon tears, recent ankle trauma and/or recent onset of symptoms (less than 3 months), failure of treatments (also intra-articular) of the involved area within the previous 3 months with steroids (systemic use or local injections), hyaluronic acid, eccentric training, and physical therapies (e.g., laser, extra-corporeal shock wave, ultrasound). Other exclusion criteria were rheumatic pathologies (rheumatoid, psoriatic and reactive arthritis, arthritis associated with inflammatory bowel diseases, and spondyloarthritis), severe systemic diseases (renal, hepatic, cardiac, infections, endocrinopathies, malignancies), immunosuppression, anticoagulants or antiaggregants therapy, hemoglobin values $<11 \mathrm{~g} / \mathrm{dL}$, and/or platelet values $<150,000 / \mathrm{mm}^{3}$.

At baseline, demographic and anthropometric data were collected. Height and weight were measured and body mass index then calculated. Pain during the previous week (both at rest and during activities) was measured by means of a 0 - to $10-\mathrm{cm}$ visual analogue scale (VAS); function was evaluated by means of the Victorian Institute of Sport Assessment-Achilles (VISA-A) questionnaire (adapted to the Italian language) [12]. The VISA-A questionnaire provides a subjective functional evaluation of the tendon and consists of 8 questions which measure the domains of pain and function in daily living and sports activity. Results range from 0 to 100 , where 100 represents the perfect score.

Before treatment, patients underwent an US-CD evaluation, using a high-resolution, multi-frequency $(6-15 \mathrm{MHz})$ linear array transducer (ProSound ALPHA10, Aloka, Japan). According to a standard protocol [13], both longitudinal and transverse scans were taken with the patient lying prone, with the feet hanging over the edge of the table at $90^{\circ}$ of flexion. The presence of dishomogeneous hypo- or hyperechoic thickening, diffuse or focal, of the tendon, associated with loss of the normal fibrillary pattern and/or irregularity of the tendon margins, was considered as a sign of degeneration. According to an arbitrary classification, based on the structural abnormalities [14], tendons were then stratified for severity as "mild" (one area of disorganized echotexture, i.e., focal dishomogeneous area with loss of fibrillary pattern), "moderate" (some areas of disorganized echotexture, i.e., dishomogeneous hypo- or hyperechoic tendon damage with altered fibrillary pattern), and "severe" (diffuse disorganized echotexture and hypo- or hyperechoic areas with irregularity of tendon margins and/or calcifications) [14].

The presence of neovascularization was investigated by means of $\mathrm{CD}$ and graded as $0,+1,+2$, and +4 according to a semi-quantitative estimate of the number of vessels. When no vessels were visible, the estimation was 0 . When there were 1 or 2 small vessels mostly in the anterior part of the tendon, the estimation was +1 . When there were several irregular vessels throughout the tendon, the estimation was +2 to $+4[15,16]$. To avoid artifacts, sensitivity was optimized for low flow, and the gain was set just below the noise level.

After clinical and instrumental evaluation, the subjects were submitted to PRP treatment performed in sterile conditions and under US guidance. PRP was prepared using the Regen Lab A-PRP Kit (Regen Lab, Switzerland). $8 \mathrm{~mL}$ of autologous blood was harvested from the cubital vein and collected into a tube containing a citrate anticoagulant in addition to the thixotropic cell separation gel. The 
Table 1. PRP diffusion in patients with mid-portion Achilles tendinopathy according to the severity of tendon lesions

\begin{tabular}{|c|c|c|c|c|}
\hline & Total & Mild & Moderate & Severe \\
\hline Number of tendons treated & 40 & 15 & 15 & 10 \\
\hline Longitudinal & 40 & 15 & 15 & 10 \\
\hline Cross-sectional & 9 & 2 & 3 & 4 \\
\hline Along the paratenon & 4 & 1 & 1 & 2 \\
\hline \multicolumn{5}{|c|}{ Distance from the tip of the needle } \\
\hline Mean, mm & $34.5 \pm 17.7$ & $21 \pm 9.9$ & $35.2 \pm 11.5^{*}$ & $53.5 \pm 17.3^{\dagger, 末}$ \\
\hline Min.-max., mm & $9-73$ & $9-38$ & $16-51$ & $25-73$ \\
\hline
\end{tabular}

$*$ Moderate vs. mild, $p=0.005 .{ }^{\dagger}$ Severe vs. mild, $p=0.0000 .{ }^{*}$ Severe vs. moderate, $p=0.004$.

tube was carefully turned upside down 5 times to homogenize the blood with the anticoagulant. After centrifugation (single spin, force [relative centrifugal force]: $1,500 \mathrm{~g}, 3,400 \mathrm{rpm}$ for $5 \mathrm{~min}$ ), the blood was fractionated, with the red blood cells trapped under the gel and the cellular sediment, including the platelets, settled on the surface of the gel. Therefore, by gently inverting the tube several times, the sediment was resuspended in the plasma supernatant and PRP $(1.6 \times$ native platelet concentration, $>80 \%$ platelet recovery, no leukocytes, red blood cell remnant $<0.3 \%$, as mean $)$ was obtained. PRP $(4-5 \mathrm{~mL})$ was then collected into a Luer-Lock syringe and was ready for use. On the whole, 3 injections were performed at intervals of 2 weeks.

After peritendinous anesthesia with $5 \mathrm{~mL}$ of mepivacaine $2 \%$, a 21-G needle was inserted into the degenerate tendon and small autologous PRP amounts (for a total of 4-5 mL) were left at the site of the most damaged areas. When the amount of PRP in the diseased areas was judged insufficient, the needle was moved slightly and a new PRP injection was made nearby.

Under US control, PRP distribution was assessed in real time visualizing the material in the tendon; in the following 10-15 min, further evaluations were performed at regular intervals. During this period, patients were instructed not to move the leg or feet until additional US-CD scans were completed.

The following parameters were registered for all patients: (1) the longitudinal and cross-sectional intratendinous diffusion; (2) the maximal distance reached by PRP (in $\mathrm{mm}$ ) from the tip of the needle; (3) the possible distribution along the paratenon; (4) the possible leakage into the surrounding soft tissues (e.g., Kager fat pad); (5) the presence of neovascularization evaluated by means of $\mathrm{CD}$ and graded according to the semi-quantitative estimate of the number of vessels reported above. The PRP diffusion was classified as small $(<30 \mathrm{~mm})$ or large $(>30 \mathrm{~mm})$. These cutoff values have already been established.

Patients were discharged from the unit after $30 \mathrm{~min}$. They were advised to refrain from strenuous activities for at least 3-4 days; ice packs and analgesic (but not NSAIDs) were allowed. Moreover, patients were asked to register possible side effects, such as pain, swelling, heat, functional limitations, and analgesic consumption, during the following days. After the second procedure, patients were submitted to a rehabilitation program. Eccentric training and stretching [17] was recommended daily ( 3 sets $\times 15$ repetitions) at least for 3 months, during which a gradual return to sport activities (when practiced) was encouraged.

Clinical assessments were repeated at 3 and 6 months after the last injection along with the VAS and VISA-A scores. The VISA-A score differences between the values registered at baseline and after 3 and 6 months were calculated. Using this parameter, the response to treatment was classified as unsatisfactory (increase from 0 to 9 ), moderate (10-20), and satisfactory $(>20)$. These values were assumed because previous research showed that an increase of 10 or more points is needed to appreciate a clinical change [18, 19].

Parameters of PRP diffusion were registered in the 3 subgroups of patients with mild, moderate, and severe lesions as seen on US. The values of clinical parameters registered at 3 and 6 months were compared with baseline values in all subjects and in the subjects with small or large PRP diffusion ( $<30$ or $>30 \mathrm{~mm}$ from the tip of the needle). Data are reported as means \pm standard deviations for continuous variables, whereas categorical and dichotomous variables are reported as frequencies and percentages. The two-sample Student's $t$ test was used to compare continuous variables when the distribution of data was normal; the Wilcoxon rank sum test was used otherwise. The $\chi^{2}$ test was used to evaluate associations between categorical data. The significance level was determined at $p<0.05$.

\section{Results}

Forty patients with mid-portion Achilles tendinopathy ( 24 males, 16 females, mean age $34.5 \pm 9.8$, body mass index $24.2 \pm 3.4$ ) were treated. PRP diffusion inside and outside the tendons is shown in Table 1. The diffusion inside the tendon was longitudinal in all cases and crosssectional only in 9 of the 40 cases. In 4 cases, PRP was found in the paratenon and in 3 cases outside the tendon. The mean distance from the tip of the needle increased significantly according to the severity of tendon damage. 
Table 2. Neovessels in patients with mid-portion Achilles tendinopathy according to the severity of tendon lesions before and after PRP injection

\begin{tabular}{|c|c|c|c|c|c|c|c|c|}
\hline & \multicolumn{4}{|c|}{ Baseline } & \multicolumn{4}{|c|}{ After injection } \\
\hline 0 & 13 & 8 & 5 & 0 & 26 & 13 & 10 & 3 \\
\hline+1 & 13 & 6 & 4 & 3 & 6 & 2 & 2 & 2 \\
\hline+4 & 1 & 0 & 0 & 1 & 1 & 0 & 0 & 1 \\
\hline
\end{tabular}

Table 3. Outcomes of PRP treatment in all patients with mid-portion Achilles tendinopathy

\begin{tabular}{lcclll}
\hline & Baseline & 3 months & $p$ & 6 months & $p$ \\
\hline VAS at rest & $2.4 \pm 1.1$ & $1.3 \pm 0.8$ & 0.000 & $0.9 \pm 0.7$ & 0.000 \\
VAS activities & $5.5 \pm 1$ & $3.1 \pm 1.9$ & 0.000 & $2.6 \pm 2.2$ & 0.000 \\
VISA-A & $48.9 \pm 7.2$ & $61.1 \pm 12.7$ & 0.000 & $68.2 \pm 14.1$ & 0.000 \\
\hline Outcome, $n$ (\%) & & & & \\
$\quad$ Satisfactory & $8(20)$ & $15(37.5)$ & $\mathrm{ns}$ \\
$\quad$ Intermediate & $17(42.5)$ & $17(42.5)$ & $\mathrm{ns}$ \\
$\quad$ Unsatisfactory & $15(37.5)$ & $8(20)$ & $\mathrm{ns}$ \\
\hline
\end{tabular}

Values are means \pm standard deviations unless otherwise indicated. ns, not significant.

Table 4. Outcomes of the treatment in the patients with Achilles tendinopathy according to the PRP diffusion in the tendon

\begin{tabular}{lcrc}
\hline & $\begin{array}{l}\text { Diffusion } \\
<30 \mathrm{~mm}\end{array}$ & \multicolumn{1}{c}{$\begin{array}{c}\text { Diffusion } \\
>30 \mathrm{~mm}\end{array}$} & $p$ \\
\hline Tendons, $n$ & 18 & 22 & \\
VISA-A, mean \pm SD & & & \\
$\quad$ Baseline & $50.7 \pm 8.5$ & $47.4 \pm 5.7$ & 0.1 \\
3 months & $62.8 \pm 15$ & $59.6 \pm 10.7$ & 0.4 \\
$\quad$ months & $71.2 \pm 14.1$ & $65.7 \pm 13.9$ & 0.2 \\
Outcomes at 6 months, $n(\%)$ & & & \\
$\quad$ Satisfactory & $8(44.4)$ & $7(31.8)$ & $\mathrm{ns}$ \\
$\quad$ Intermediate & $7(38.8)$ & $10(45.4)$ & $\mathrm{ns}$ \\
$\quad$ Unsatisfactory & $3(16.6)$ & $5(22.7)$ & $\mathrm{ns}$ \\
\hline
\end{tabular}

$\mathrm{SD}$, standard deviation; ns, not significant.
Neovessels were observed at baseline in 7/15 (46,6\%), $10 / 15(66.6 \%)$, and 10/10 (100\%) patients with mild, moderate, and severe lesions, respectively. After injection (10-15 $\mathrm{min}$ ), these percentages were significantly reduced (in total 14 vs. $27, p=0.003$ ) (Table 2). VAS mean scores decreased and VISA-A mean scores increased at 3 and 6 months of follow-up. The number of satisfactory outcomes (as previously defined) was $8 / 40(20 \%)$ and $15 / 40(37.5 \%)$ at 3 months and 6 months, respectively (Table 3 ). No significant differences were found between the subjects classified according to the PRP diffusion along the tendon (Table 4).

\section{Discussion}

The results of the present study show that PRP spread along the tendon in all cases. The diffusion was longitudinal in the proximal-distal direction and was associated with a simultaneous bulging of the tendon. In some cases, especially in the most damaged tendons, an intratendinous cleft was produced by the injected substance and appeared as an anechoic area with irregular margins. The distance reached by PRP increased progressively with the severity of the US lesions. A cross-sectional diffusion was observed only in few cases as well as to the paratenon and/ or outside the tendon. Neovessels in the damaged tendons in the majority of cases disappeared after a short while because of the pressure of the injected fluid.

Our study is unique as the PRP injections were performed in vivo on a large tendon with sonographic features of different/increasing severity. Previous studies have either looked at PRP injection in cadavers or smaller tendons of the elbow. The study by Wiegerinck et al. [7] was performed in cadavers with normal Achilles tendons, and the distance reached by the fluid from the site of injection was 
not evaluated. In the studies by Loftus et al. [9] and Park et al. [10] performed in vivo, a diffusion outside the tendon was observed more frequently than in our experiments, but this can be explained by the smaller dimensions of tendons (e.g., elbow tendons) and the pressure and amount of fluid injected. The new finding of our study concerns the relationship between PRP diffusion and clinical outcomes. In this regard, we did not observe any difference between the patients in whom the substance spread within a short distance and the ones in whom a wide dispersion of the product inside the tendon was noticed.

Theoretically, several factors can influence PRP distribution into a degenerated tendon; the anatomy of the tendon itself, the severity of lesions, the visco-elastic properties of the fluid, its amount, and the site and pressure of the injection. In the more damaged areas, the diffusion is influenced by the characteristics of the lesions; mucoid or fatty degeneration may be associated with a disorganized collagen structure and/or fibril tears. Moreover, vacuoles or calcifications may be present, and fenestrations may be caused by the needle. Therefore, the different firmness of these tissues and their architectural organization influence the amount of PRP which spreads along the tendon, because it is conceivable that fluids may move easily through less solid tissues. The longitudinal diffusion of PRP can be ascribed to the unsticking of the inter-fibrillary septa, because in chronic Achilles tendinopathy the collagen is loose and weak and, therefore, more prone to tear. Indeed, the distance reached by PRP increased progressively with the severity of the US lesions.

Despite this large variability of distribution, which is due to unforeseeable individual anatomical situations, the outcomes were similar in all groups with different diffusion of the injectate along the tendon. Thus, the theoretical assumption that in case of a large diffusion it is unlikely that PRP can develop therapeutic effects is not justified. Indeed, the efficacy of PRP in the treatment of tendon disorders in the short term is related to the ability of several molecules (growth factors, chemokines, signaling proteins), stored inside platelets, to modulate different aspects of tendon repair (inflammatory response, chemotaxis, angiogenesis, cell migration and proliferation), whereas in the long term, the activation of stem/progenitor cells plays a major role in the regeneration of tendon-like tissues [4, $5,20,21]$. Therefore, instead of the amount of PRP retained near the more damaged areas, the presence of vital cells which can respond to the stimulation is more important. On the other hand, as previously suggested [22], the tendon must be considered as a functional unit, so that we can hypothesize that the positive changes of metabolism which occur also at a distance from the more injured areas may influence the whole structure [23].

The present study has several strengths. A standard procedure was adopted in all cases, such as US evaluation and PRP injection performed by the same operator, use of the same anesthetic drug, and implementation of an eccentric exercise program in the follow-up. However, some limitations must be acknowledged: the small number of patients included in each group, the short follow-up period, the arbitrary definitions of small or large PRP diffusion as well as the arbitrary classification of the structural abnormalities, also taking into account the lack of equivalence between the US features of tendon degeneration and the morphology of tissues in pathologic conditions. Finally, it must be noted that several factors can influence the therapeutic outcome. First, it is very difficult to quantify the level of physical activity and the compliance of each patient to the rehabilitation protocol; second, a placebo effect, which usually ranges from 15 to $30 \%$, although reasonably similar in all groups, cannot be ruled out.

\section{Conclusions}

Our results show that there is no clinical difference between cases in which PRP diffuses to a greater extent and those in which it does not. In other words, the diffusion of PRP far from the damaged areas of pathologic tendons is of limited relevance, whereas the metabolic stimulation of the whole tendon is more important. This reinforces the validity of the peppering technique which allows the distribution of PRP over a wide region of the anatomical structure, considered as a functional unit. Moreover, this suggests that further research is required with respect to the efficacy of PRP versus other treatment options.

\section{Statement of Ethics}

This study was performed in accordance with the ethical standards as laid down in the 1964 Declaration of Helsinki and its later amendments. Informed written consent for participation was obtained from each patient. Institutional ethics committee approval was not required as this was an observational study [11] and because the PRP procedure is safe and currently performed in routine clinical practice.

\section{Disclosure Statement}

The authors declare no conflicts of interest. 


\section{References}

1 Filardo G, Di Matteo B, Kon E, Merli G, Marcacci $M$. Platelet-rich plasma in tendon-related disorders: results and indications. Knee Surg Sports Traumatol Arthrosc. 2018 Jul; 26(7):1984-99.

2 Boesen AP, Hansen R, Boesen MI, Malliaras $\mathrm{P}$, Langberg $\mathrm{H}$. Effect of high-volume injection, platelet-rich plasma, and sham treatment in chronic midportion Achilles tendinopathy: a randomized double-blinded prospective study. Am J Sports Med. 2017 Jul; 45(9):2034-43

3 Abate M, Schiavone C, Salini V, Andia I. Revisiting physical examination: abadie's sign and Achilles intratendinous changes in subjects with diabetes. Med Princ Pract. 2014; 23(2):186-8.

4 Abate M, Di Gregorio P, Schiavone C, Salini $\mathrm{V}$, Tosi U, Muttini A. Platelet rich plasma in tendinopathies: how to explain the failure. Int J Immunopathol Pharmacol. 2012 Apr-Jun; 25(2):325-34.

5 Andia I, Abate M. Platelet-rich plasma: underlying biology and clinical correlates. Regen Med. 2013 Sep;8(5):645-58.

6 Wilson JJ, Lee KS, Chamberlain C, DeWall R, Baer GS, Greatens M, et al. Intratendinous injections of platelet-rich plasma: feasibility and effect on tendon morphology and mechanics. J Exp Orthop. 2015 Dec;2(1):5.

7 Wiegerinck JI, de Jonge S, de Jonge MC, Kerkhoffs GM, Verhaar J, van Dijk CN. Comparison of postinjection protocols after intratendinous Achilles platelet-rich plasma injections: a cadaveric study. J Foot Ankle Surg. 2014 Nov-Dec;53(6):712-5.
8 Abate M, Verna S, Di Gregorio P, Salini V, Schiavone C. Sonographic findings during and after Platelet Rich Plasma injections in tendons. Muscles Ligaments Tendons J. 2014 May;4(1):29-34.

9 Loftus ML, Endo Y, Adler RS. Retrospective analysis of postinjection ultrasound imaging after platelet-rich plasma or autologous blood: observational review of anatomic distribution of injected material. AJR Am J Roentgenol. 2012 Oct;199(4):W501-5.

10 Park GY, Kwon DR, Cho HK, Park J, Park JH. Distribution of Platelet-rich Plasma after Ultrasound-Guided Injection for Chronic Elbow Tendinopathies. J Sports Sci Med. 2017 Mar;16(1):1-5.

11 Kıraç FS. Is ethics approval necessary for all trials? A clear but not certain process. Mol Imaging Radionucl Ther. 2013 Dec;22(3):73-5.

12 Maffulli N, Longo UG, Testa V, Oliva F, Capasso $\mathrm{G}$, Denaro $\mathrm{V}$. Italian translation of the VISA-A score for tendinopathy of the main body of the Achilles tendon. Disabil Rehabil. 2008;30(20-22):1635-9.

13 Sunding K, Fahlström M, Werner S, Forssblad M, Willberg L. Evaluation of Achilles and patellar tendinopathy with greyscale ultrasound and colour Doppler: using a four-grade scale. Knee Surg Sports Traumatol Arthrosc. 2016 Jun;24(6):1988-96.

14 Salini V, Abate M. Percutaneous steroidal treatment in relapses of chronic tendinopathies: a pilot study. Int J Immunopathol Pharmacol. 2011 Jan-Mar;24(1):211-6.

15 Tol JL, Spiezia F, Maffulli N. Neovascularization in Achilles tendinopathy: have we been chasing a red herring? Knee Surg Sports Traumatol Arthrosc. 2012 Oct;20(10):1891-4.
16 Ohberg L, Lorentzon R, Alfredson H. Neovascularisation in Achilles tendons with painful tendinosis but not in normal tendons: an ultrasonographic investigation. Knee Surg Sports Traumatol Arthrosc. 2001 Jul;9(4):233-8.

17 Malliaras P, Barton CJ, Reeves ND, Langberg $\mathrm{H}$. Achilles and patellar tendinopathy loading programmes : a systematic review comparing clinical outcomes and identifying potential mechanisms for effectiveness. Sports Med. 2013 Apr;43(4):267-86.

18 Pham T, van der Heijde D, Altman RD, Anderson JJ, Bellamy N, Hochberg $\mathrm{M}$, et al. OMERACT-OARSI initiative: osteoarthritis Research Society International set of responder criteria for osteoarthritis clinical trials revisited. Osteoarthritis Cartilage. 2004 May; 12(5):389-99.

19 Macdermid JC, Silbernagel KG. Outcome evaluation in tendinopathy: foundations of assessment and a summary of selected measures. J Orthop Sports Phys Ther. 2015 Nov; 45(11):950-64.

20 Andia I, Maffulli N. Muscle and tendon injuries: the role of biological interventions to promote and assist healing and recovery. Arthroscopy. 2015 May;31(5):999-1015.

21 D’Addona A, Maffulli N, Formisano S, Rosa D. Inflammation in tendinopathy. Surgeon. 2017 Oct;15(5):297-302.

22 Maffulli N, Testa V, Capasso G, Bifulco G, Binfield PM. Results of percutaneous longitudinal tenotomy for Achilles tendinopathy in middle- and long-distance runners. Am J Sports Med. 1997 Nov-Dec;25(6):835-40.

23 de Vos RJ. Does platelet-rich plasma increase tendon metabolism? Adv Exp Med Biol. 2016; 920:263-73. 